\title{
De novo pure erythroid leukemia: refining the clinicopathologic and cytogenetic characteristics of a rare entity
}

\author{
Erica F Reinig ${ }^{1}$, Patricia T Greipp ${ }^{2}$, April Chiu ${ }^{1}$, Matthew T Howard ${ }^{1}$ and \\ Kaaren K Reichard ${ }^{1}$ \\ ${ }^{1}$ Division of Hematopathology, Department of Laboratory Medicine and Pathology, Mayo Clinic, Rochester, \\ MN, USA and ${ }^{2}$ Division of Laboratory Genetics and Genomics, Department of Laboratory Medicine and \\ Pathology, Mayo Clinic, Rochester, MN, USA
}

\begin{abstract}
Per the revised fourth edition World Health Organization classification of acute myeloid leukemia, pure erythroid leukemia is now the sole type of acute erythroid leukemia. The diagnosis of this rare entity is often challenging and the cytologic overlap with non-neoplastic (eg, megaloblastic anemia) and neoplastic entities (eg, other types of acute leukemia and non-hematopoietic malignancies) warrants a significant degree of clinical, laboratory, immunophenotypic, and genetic investigation. Given the limited number of reports of this rare and diagnostically challenging entity, we report detailed clinicopathologic characteristics from 15 patients, the largest series thus far, of primary de novo pure erythroid leukemia to provide further diagnostic insights into this entity and reveal strategies for making the diagnosis. We found that de novo pure erythroid leukemia is a disease of adults (median age 68 years), exhibits a striking male predominance, is universally associated with an abnormal karyotype and has an exceedingly poor overall median survival of $\mathbf{1 . 4}$ months. Given the general inability of immunophenotypic markers to discriminate neoplastic from non-neoplastic erythroid proliferations, key features identified in this study to help establish the diagnosis of pure erythroid leukemia and exclude mimickers include circulating pronormoblasts, clear-cut dysplasia in erythroid, granulocytic, and/or megakaryocytic lineage, utilization of a broad immunophenotypic panel, TP53 immunohistochemical positivity, and identification of a complex, often highly complex, karyotype. Given the gravity of a diagnosis of de novo pure erythroid leukemia, it should be rendered with utmost confidence.
\end{abstract}

Modern Pathology (2018) 31, 705-717; doi:10.1038/modpathol.2017.175; published online 12 January 2018

The 2016 revised World Health Organization classification of hematological malignancies recognizes pure erythroid leukemia as the sole type of acute leukemia with bona fide erythroid differentiation. ${ }^{1,2}$ In the prior 2001 and 2008 World Health Organization classifications, erythroleukemia (erythroid/myeloid) and pure erythroid leukemia were recognized as subtypes of the broader category of acute erythroid leukemia. ${ }^{1-3}$ However, the former has been removed as a diagnostic category from the revised fourth edition World Health Organization classification because of the low reproducibility of non-erythroid blast counts and the close biological relationship

Correspondence: Dr KK Reichard, Division of Hematopathology, Department of Laboratory Medicine and Pathology, Mayo Clinic, 200 1st St. Hilton Building 8-00C, Rochester, MN 55902, USA.

E-mail: reichard.kaaren@mayo.edu

Received 15 August 2017; revised 16 October 2017; accepted 17 October 2017; published online 12 January 2018 between erythroleukemia and myelodysplastic syndrome based on findings from multiple, large comprehensive studies. ${ }^{1-11}$

Pure erythroid leukemia is a rare and often challenging diagnosis. The diagnosis is typically difficult for several reasons, including morphologic similarity of pronormoblasts with other cell types, CD34 negativity in the erythroblasts, and weak to absent immunoreactivity for the erythroid-specific markers glycophorin A and hemoglobin. Given the dismal prognosis in pure erythroid leukemia, it is critical to exclude neoplastic differential diagnostic considerations, which may be associated with a more favorable prognosis, and non-neoplastic conditions. Potential diagnostic pitfalls for pure erythroid leukemia include megaloblastic anemia, hemolytic anemia, other subtypes of acute myeloid leukemia, myeloid neoplasms with abundant erythroid precursors, and non-hematopoietic malignancies such as carcinoma and sarcoma. While a 
specific cytogenetic and/or molecular abnormality has not yet been described for pure erythroid leukemia, cytogenetic studies have shown that a complex karyotype is almost invariably encountered in these cases. ${ }^{1,7,12-21}$ In addition to the morphologic overlap, pure erythroid leukemia occasionally shows hyperdiploidy/near tetraploidy by cytogenetic studies, karyotypically mimicking a carcinoma or sarcoma, which can further complicate the final interpretation.

Given the significant diagnostic challenges and associated poor prognosis of pure erythroid leukemia, it is important to be aware of and exclude all potential differential diagnostic considerations. The majority of our knowledge about pure erythroid leukemia stems from multiple large comprehensive studies on erythroleukemia (erythroid/myeloid), and de novo and secondary pure erythroid leukemia. In order to gain further insights specifically into de novo pure erythroid leukemia and help inform diagnostic decision-making, we sought to investigate the clinicopathologic and cytogenetic characteristics of de novo pure erythroid leukemia cases from our institutional experience. To our knowledge, this is the largest series to date reporting on de novo pure erythroid leukemia.

\section{Materials and methods}

\section{Case Selection}

Bone marrow cases diagnosed as pure erythroid leukemia according to the revised fourth edition World Health Organization criteria were retrieved from the Mayo Clinic Rochester Department of Laboratory Medicine and Pathology files during the period from January 2001 to January 2017.1,2 Pure erythroid leukemia cases that were ultimately included were restricted to those determined to be de novo in presentation based on a rigorous review of clinical and pathologic material. Cases with a prior history of a myeloid neoplasm and/or exposure to chemotherapy and/or radiation were excluded. Within the time frame of the study, 44 total cases were diagnosed as erythroleukemia. Eleven of the 44 were diagnosed as pure erythroid leukemia but were

Table 1 Inclusion criteria for de novo (primary) pure erythroid leukemia cases ${ }^{\mathrm{a}}$

- 2016 World Health Organization criteria for a diagnosis of pure erythroid leukemia are met: $>80 \%$ immature erythroid precursors with $\geq 30 \%$ proerythroblasts (adapted from ref. 1)

- No history of an antecedent myeloid neoplasm

- No prior history of chemotherapy and/or radiotherapy exposure

- Absence of a recurring genetic abnormality that would satisfy classification as an alternative World Health Organizationdefined subtype of acute myeloid leukemia

${ }^{\mathrm{a}}$ All four of these stated criteria were met in order to be included in the current case cohort. excluded either because they were secondary to prior chemotherapy and/or radiotherapy (ie, therapy-related myeloid neoplasm, $N=6$ ) or arose from an antecedent myelodysplastic syndrome $(N=4)$ or myeloproliferative neoplasm $(N=1)$ without prior therapy. We also excluded 18 cases of erythroleukemia, erythroid/myeloid type (M6a) as this subtype of acute erythroid leukemia has been eliminated from the revised fourth edition of World Health Organization classification. Table 1 summarizes the inclusion criteria for the cases in this study. This study was approved by the Mayo Clinic Institutional Review Board.

\section{Clinical Data}

Key clinical data obtained from the medical record included patient age, gender, and clinical course, including time to death and overall survival.

\section{Morphologic Studies}

Diagnoses were established according to the revised fourth edition World Health Organization classification criteria using a combination of morphologic, immunohistochemical, and/or molecular findings. ${ }^{1,2}$ All peripheral blood, bone marrow aspirates, trephine biopsies were reviewed by an experienced hematopathologist (KKR). The diagnostic criteria were met by bone marrow aspirate and/or core biopsy findings.

\section{Immunophenotyping Studies}

Immunohistochemistry. Immunohistochemical analysis was performed on $5 \mu \mathrm{m}$, formalin-fixed, paraffin-embedded sections in all cases. A broad immunohistochemical panel was utilized in the evaluation of the cases. Primary antibodies included CD34 (QBEnd/10, Leica (Novocastra)), CD117 (YR145, Cell Marque), CD71 (MRQ-48, Cell Marque), E-Cadherin (4A2C7, Life Technologies), Myeloperoxidase (Dako), Hemoglobin (Cell Marque), Glycophorin A (GA-R2, Ventana), and TP53 (DO-7, Ventana).

Flow cytometry. After isotonic erythrocyte lysis, flow cytometric immunophenotyping was performed on anticoagulated bone marrow aspirate specimens using previously described methods. ${ }^{22}$ Samples were examined with flow cytometric immunophenotyping using a single eight-color tube containing antibodies from BD Biosciences (San Jose, CA; CD13-PE (L138), CD15-V450 (MMA), CD16-APC-H7 (3G8), CD33-PE-Cy7 (P676), CD34-PerCP-Cy5.5 (8G12), CD45-V500 (H130), HLA-DR-FITC (L243), and CD117-APC (104D2)). A total of 100000 events were collected per case. The data were analyzed using Kaluza software (Beckman-Coulter, Brea, CA) and/or Diva software (BD Biosciences). 


\begin{tabular}{|c|c|c|c|c|c|c|c|c|c|}
\hline Case & $\begin{array}{c}\text { Age } \\
\text { (years) }\end{array}$ & Gender & $\begin{array}{l}\text { Peripheral } \\
\text { blood: } \\
\text { pancytopenia }\end{array}$ & $\begin{array}{l}\text { Peripheral blood: } \\
\text { bicytopenia (anemia and } \\
\text { thrombocytopenia) }\end{array}$ & $\begin{array}{l}\text { Peripheral blood: } \\
\text { circulating } \\
\text { erythroblasts (\%) }\end{array}$ & $\begin{array}{c}\text { Bone } \\
\text { marrow: } \\
\text { cellularity } \\
(\%)\end{array}$ & $\begin{array}{c}\text { Bone marrow: \% red blood } \\
\text { cell precursors (not } \\
\text { pronormoblasts)/\% } \\
\text { pronormoblasts (based on } \\
\text { aspirate or core) }\end{array}$ & $\begin{array}{l}\text { Bone marrow: } \\
\% \text { myeloid } \\
\text { blasts of total } \\
\text { nucleated } \\
\text { elements }^{a}\end{array}$ & $\begin{array}{l}\text { Immunophenotypic } \\
\text { characteristics of the myeloid } \\
\text { (non-erythroid) blasts } \\
\text { (determined by flow } \\
\text { cytometry); positive for }\end{array}$ \\
\hline 1 & 61 & M & + & & None & 95 & 17/67 (aspirate) & $<1$ & $\begin{array}{l}\text { CD45 (dim), CD34, CD117, } \\
\text { CD13, CD33, CD7, HLA-DR }\end{array}$ \\
\hline 2 & 57 & M & + & & None & 80 & 30/55 (core) & $<5$ & $\begin{array}{l}\text { CD45 (dim), CD34, CD117, } \\
\text { CD13, CD33, HLA-DR }\end{array}$ \\
\hline 3 & 78 & $\mathrm{M}$ & & WBC 7.6 & 4 & 90 & 0/85 (core) & $<2$ & No myeloblasts detected \\
\hline 4 & 70 & $\mathrm{M}$ & & WBC 6.4 & 5 & 95 & 20/70 (core) & $<2$ & $\begin{array}{l}\text { CD } 45 \text { (dim), CD117, CD13, } \\
\text { CD33, CD38, MPO }\end{array}$ \\
\hline 5 & 68 & $\mathrm{~F}$ & & WBC 5.9 & 1 & 50 & 50/31 (aspirate) & 3 & $\begin{array}{l}\text { CD45 (dim), CD34, CD117, } \\
\text { CD13, CD33, HLA-DR, CD38 }\end{array}$ \\
\hline 6 & 47 & $\mathrm{~F}$ & & WBC 4.3 & Rare & 70 & 3/81 (aspirate) & $<1$ & No myeloblasts detected \\
\hline 7 & 62 & $\mathrm{M}$ & + & & Rare & 95 & 20/70 (core) & Rare & No myeloblasts detected \\
\hline 8 & 68 & $\mathrm{M}$ & $r$ & WBC $32.6^{\mathrm{b}}$ & None & 70 & $40 / 50$ ( core) & $<5$ & Flow only performed for CLL \\
\hline 9 & 80 & $\mathrm{M}$ & + & & 5 & 80 & 0/90 (core) & Rare & No myeloblasts detected \\
\hline 10 & 78 & M & + & & 61 & 95 & 31/59 (aspirate) & 5 & $\begin{array}{l}\text { CD45 (dim), CD34, CD117, } \\
\text { CD13, HLA-DR, CD38 }\end{array}$ \\
\hline 11 & 87 & M & NA & & Rare & 90 & 0/81 (aspirate) & 1 & $\begin{array}{l}\text { CD45 (dim), CD34, CD117, } \\
\text { CD33, HLA-DR, CD38 }\end{array}$ \\
\hline 12 & 60 & M & + & & None & 70 & 27/60 (aspirate) & 2 & $\begin{array}{l}\text { CD45 (dim), CD34, CD117, } \\
\text { CD13 (partial), CD33 (partial), } \\
\text { HLA-DR, CD38 }\end{array}$ \\
\hline 13 & 79 & M & + & & 3 & 50 & 15/75 (core) & Rare & $\begin{array}{l}\text { CD45 (dim), CD34, CD13, } \\
\text { CD33, CD117, HLA-DR, CD38 }\end{array}$ \\
\hline 14 & 70 & $\mathrm{~F}$ & & WBC 6.4 & Rare & 95 & 50/40 (core) & $<5$ & Flow not performed \\
\hline 15 & 60 & $\mathrm{M}$ & & WBC 6.3 & None & 95 & 1/84 (aspirate) & 0 & No myeloblasts detected \\
\hline
\end{tabular}

F, female; M, male; NA, not available; WBC, white blood cell count.

${ }^{a}$ In cases of core biopsies, CD34 immunohistochemistry was utilized.

${ }^{\mathrm{b}}$ Concurrent CLL (75\% lymphocytes). 
Table 3 Immunohistochemical, cytogenetic/FISH features, and clinical outcome of de novo pure erythroid leukemia

\begin{tabular}{|c|c|c|c|c|c|c|c|c|c|c|}
\hline$P t$ & CD34 & $C D 71$ & $C D 117$ & E-Cadherin & $C D 61$ & p53 & Glycophorin A & Hemoglobin & Karyotype & $\begin{array}{l}\text { Time to death } \\
\text { (in months) }\end{array}$ \\
\hline 1 & - & + & - (flow) & + & - & ND & ND & + & $\begin{array}{l}57-66, \mathrm{XY},+\operatorname{der}(\mathrm{Y} ; 15)(\mathrm{q} 10 ; \mathrm{q} 10),+1, \operatorname{inv}(1)(\mathrm{p} 22 \mathrm{q} 25) \times 2,+2,+3, \operatorname{add}(3)(\mathrm{p} 13) \times 2, \\
+4,+6,+6,-7,+8,+8,+14, \operatorname{add}(15)(\mathrm{p} 11.2),+18,+18,+19,+19,+20,+20,+21 \\
+\operatorname{add}(21)(\mathrm{p} 11.2),-22,+0-2 \mathrm{r},+1-7 \operatorname{mar}[\mathrm{cp} 16] / 46, \mathrm{XY}, \operatorname{del}(9)(\mathrm{q} 13 \mathrm{q} 22)[4]\end{array}$ & 2.10 \\
\hline 2 & - & + & + & + & - & + & + (weak subset) & $\begin{array}{l}\text { Not } \\
\text { interpretable }\end{array}$ & $\begin{array}{l}\text { 43,XY,add(1)(p36.1),del(4)(q21q31.3), - 5,t(5;12)(p13;q15),del(7)(q22q34), } \\
\text { dic(8;13)(p23;p12), - 9, - 12,-13,-15, - 16,-17,psu dic(18;21)(p11.2; } \\
\text { p11.2),dic(20;22)(p13;q13),+22,+4mar,+2r[1]/78-79,idemx2[cp3]/46,XY } \\
\text { [17] }\end{array}$ & 5.77 \\
\hline 3 & \pm & + & + & + & - & + & + & + & $\begin{array}{l}\text { 46,XY,add(5)(q11.2)[1]/44,XY,add(5)(q11.2),+add(6)(p25), }-7,-9,-9, \text { add } \\
\text { (12)(p11.1),add(14)(p11.2),add(15)(p11.2),-16,i(17)(q10),+der(?)t(?;7)(?; } \\
\text { q11.2)[1]/84-96,XXYY,+der(4)add(4)(p14)hsr(4)(q12),-5, - } 5 \text {,add(5) } \\
\text { (q11.2)x2[1],add(6)(p25),add(6)(q25), - 7,- 7,-9,-9,ins(9;?)(q13;?) } \\
\text { x2,-16,-16,-17,i(17)(q10),+der(?)t(?;7)(?;q11.2)x2,+0-3r,+4-9mar } \\
{[\text { cp13]/46,XY[5] }}\end{array}$ & 2.47 \\
\hline 4 & - (flow) & + & + & + & - & + & + & $+($ small subset $)$ & $\begin{array}{l}56-59, X Y,+\operatorname{add}(1)(\mathrm{q} 12), \operatorname{add}(2)(\mathrm{p} 21),+\operatorname{add}(4)(\mathrm{q} 31.1),+\operatorname{add}(5)(\mathrm{q} 11.2),+6, \text { add } \\
(7)(\mathrm{p} 11.2),+8,+8,-9,+\operatorname{add}(10)(\mathrm{q} 22),+11,-18,+\operatorname{add}(20)(\mathrm{q} 11.2),+21,+22,+3- \\
6 \mathrm{mar}[\mathrm{cp} 20]\end{array}$ & 0.30 \\
\hline 5 & - & + & + (flow) & + & ND & + & - & + (weak subset) & $\begin{array}{l}\text { 45,XX,add(1)(p13),der(2)t(1;2)(p13;q11.2), - 3, der(5)t(2;5)(q13;q13),add(7) } \\
(\mathrm{p} 11.1),-9,-9,-17,-19,+4 \operatorname{mar}[19] / 46, \mathrm{XX}[1]\end{array}$ & 0.70 \\
\hline 6 & - (flow) & + & + & + & - & + & $+($ small subset $)$ & + (small subset) & $\begin{array}{l}\text { 41-45,X, - X,hsr(6)(q21),hsr(14)(p13), - 15,+17,hsr(19)(p13.1), - 21, - 21, } \\
+1-3 \operatorname{mar}[\mathrm{cp} 6] / 43-45, \text { idem,der(X;21)(q10;q10)[cp14] }\end{array}$ & 1.30 \\
\hline 7 & - & + & + & $+^{\mathrm{a}}$ & - & + & $+($ small subset $)$ & $+($ small subset $)$ & $\begin{array}{l}\text { 39-44,XY, }-1, \operatorname{add}(2)(\mathrm{p} 11.2),-3, \mathrm{psu} \operatorname{dic}(3 ; 12)(\mathrm{p} 21 ; \mathrm{p} 13), \operatorname{add}(7) \\
(\mathrm{p} 22),-8,-9, \operatorname{add}(9)(\mathrm{q} 13),-10, \operatorname{der}(11) \mathrm{t}(1 ; 11)(\mathrm{q} 21 ; \mathrm{p} 11.2) \operatorname{ins}(11 ; ?)(\mathrm{p} 11.2 ; ?), \\
\text { add(12)(q13),-13,add(16)(q22),-17,-18,add(19)(p13.1),-21,-22,+r, } \\
+1-7 \operatorname{mar}[15] / 46, X Y[5]\end{array}$ & 1.90 \\
\hline 8 & ND & ND & ND & ND & ND & ND & ND & ND & $\begin{array}{l}71-77, \mathrm{XY},+\mathrm{Y},+1,+1,+2,+3,+4,+6,+6,+\operatorname{add}(7)(\mathrm{q} 11.2), \mathrm{hsr}(7)(\mathrm{p} 11.2),+8,+8, \\
+9,+10,+11,+\operatorname{der}(11 ; 22)(\mathrm{q} 10 ; \mathrm{q} 10),+\operatorname{add}(12)(\mathrm{q} 13),+13,+13,+14,+14,+15, \\
+16,+18,+19, \operatorname{add}(19)(\mathrm{p} 13.3),+20,+21,+21,+22,+22,+0-1 \mathrm{r},+3-8 \mathrm{mar}[\mathrm{cp} 15] / \\
39-42, \mathrm{XY},-5, \operatorname{del}(5)(\mathrm{q} 13 \mathrm{q} 33),-17,-21,+\operatorname{der}(?) \mathrm{t}(? ; 12)(? ; \mathrm{q} 13)[\mathrm{cp} 2] / 46, \mathrm{XY} \\
{[3]}\end{array}$ & 1.40 \\
\hline 9 & - & \pm & + (weak) & - & - & ND & 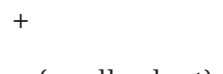 & - & $\begin{array}{l}42-44, \mathrm{X},-\mathrm{Y}, \operatorname{add}(5)(\mathrm{q} 11.2), \operatorname{add}(5)(\mathrm{q} 15), \mathrm{t}(11 ; 14)(\mathrm{p} 13 \\
\mathrm{q} 11.2),-13,-16,-17,-18, \operatorname{add}(19)(\mathrm{p} 13.3),+1-3 \mathrm{r}[\mathrm{cp} 20]\end{array}$ & 0.20 \\
\hline 10 & - & + & + & + & - & + & + (small subset) & + (small subset) & $\begin{array}{l}\text { 42-44,XY,add(1)(q32),add(2)(p13),psu dic(4;15)(q31.1;p11.2),der(5;14) } \\
\text { (q11.2;q11.2)add(5)(p13),dic(5;14)(q11.2;p11.2), - 7,add(7)(q11.2),add(11) } \\
\text { (q21),add(13)(q14), -14,add(16)(p13.1),add(17)(p13),add(17)(q21),+0-2r, } \\
\text { +0-1mar[cp20] }\end{array}$ & 1.13 \\
\hline 11 & - & + & + & + & - & ND & + (small subset) & + (small subset) & $46-47, \mathrm{X},-\mathrm{Y}, \operatorname{add}(4)(\mathrm{q} 25),-5,-17,-18,-19,+5-6 \operatorname{mar}[\mathrm{cp} 5] / 46, \mathrm{XY}[15]$ & NA \\
\hline 12 & - (flow) & + & + (flow) & ND & - & ND & $+($ small subset $)$ & + & $\begin{array}{l}\text { 52-56,X, }-\mathrm{Y},+1,+2,+\operatorname{add}(4)(\mathrm{p} 14), \text { add(5)(p13),add(5)(q11.2), }+6,+7,+8,+8, \\
\text { +10,-12, del(13) (q12q14),+14,+18,add(19)(p13.3),hsr(21)(p11.2),+add } \\
\text { (22)(p11.2),hsr(22)(p11.2),+1-2r[cp20] }\end{array}$ & 3.47 \\
\hline 13 & - & + & + & + & - & ND & ND & ND & $\begin{array}{l}\text { 43-48,XY, }- \text { Y,add(5)(q11.2), +add(11)(q13)x2, }-13,-14, \operatorname{add}(14) \\
(\mathrm{p} 11.2),-15,-16, \text { add(16)(q22), - 17,-18,add(18)(q21.1), -19,+21, dic } \\
(21 ; 21)(\mathrm{p} 13 ; \mathrm{p} 13),-22,+2-6 \mathrm{r}[\mathrm{cp} 12] / 46, \mathrm{XY}[8]\end{array}$ & 3.37 \\
\hline 14 & - & + & + & + & ND & + & + & + & $\begin{array}{l}\text { 40-47,X, - X,del(3)(q26q27),add(4)(q31),del(5)(q22q35),add(7) } \\
\text { (p14), - 9, -12, add(12)(q24), - 15,-16,del(16)(q22q24), - 17,add(17) } \\
\text { (p12), -18, -19, - 21,add(21)(p12), - 22,+1 8mar[cp16]/46,XX[4] }\end{array}$ & 0.33 \\
\hline 15 & - (flow) & + & + & + & - & + & ND & $+($ small subset $)$ & $42, X Y,-9,-10, \operatorname{der}(11 ; 15)(q 10 ; q 10),-13[15] / 84, \operatorname{idemx} 2[5]$ & NA \\
\hline
\end{tabular}

ND, not done.

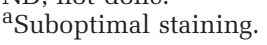


Cytogenetic and fluorescence in situ hybridization analysis. Giemsa-banded (G-banded) chromosome analysis was performed on bone marrow samples according to conventional methods. When available, at least 20 metaphases were analyzed. Karyotypes of G-banded chromosomes were described according to the 2009 International System of Human Cytogenetic Nomenclature. ${ }^{23}$ Abnormal clones were defined as two or more cells with the same structural abnormality, or the same extra chromosomes, or the presence of three or more cells with loss of the same chromosome.

Fluorescence in situ hybridization (FISH) was performed on cell suspensions prepared from fresh bone marrow aspirate pellets using standard techniques. Probes targeting TP53 to assess for deletion were utilized. FISH was performed by codenaturation on a HYBrite instrument (Vysis/Abbott, Abbott Park, IL) at a denaturation temperature of $72{ }^{\circ} \mathrm{C}$ for $2 \mathrm{~min}$ for freshly dropped cells, followed by overnight hybridization at $37^{\circ} \mathrm{C}$. At least 100 nuclei were examined for each probe whenever possible. Images were captured on a Leica DM5000B microscope (Leica Microsystems, Buffalo Grove, IL).

\section{Results}

We identified 15 cases of de novo pure erythroid leukemia. By our best estimation, pure erythroid leukemia accounts for $<1 \%$ of all acute myeloid leukemias diagnosed at our institution during the selected time frame. Given that many of the cases were received in consultation, a more precise estimate of incidence is precluded. A comprehensive summary of the clinical, laboratory, morphologic, immunophenotypic, and cytogenetic features from the cases is shown in Tables 2 and 3.

\section{Clinical Characteristics and Overall Survival}

There was a striking male predominance with a male:female ratio of 12:3. The mean patient age at diagnosis was 68 years (range 47-87). For the 13 patients with survivorship data, the average time to death was 2.0 months (range $0.2-5.77$ ) with a median overall survival of 1.4 months.

\section{Laboratory and Morphologic Findings}

Peripheral blood. Fourteen of the 15 cases had an available complete blood cell count. Seven of the 14 cases $(50 \%)$ were characterized by pancytopenia (Table 2). The remaining 7 cases had bicytopenia consisting uniformly of a combination of anemia and thrombocytopenia. On review of the peripheral blood morphology, circulating pronormoblasts were noted in 10 cases (67\%), generally comprising $5 \%$ or less of nucleated white blood cells (Figure 1(1a), (2a), and (3a)).
Bone marrow aspirate. Bone marrow aspirates/ touch preparations were reviewed in all cases. The bone marrow differential counts were determined from the aspirate smears in cases with adequate cellularity and representation (Table 2). In 8 of 15 cases, we noted discrepancies between the pronormoblast counts in the aspirate (underestimated) and core biopsy.

Myeloid blasts did not exceed $20 \%$ in any of the cases and no Auer rods were identified. The morphologic features of the pronormoblasts were similar across the cases. Wright-Giemsa-stained slides showed a proliferation of large immature cells with round/oval nuclear contours, dispersed chromatin, 1-3 elongated nucleoli, and a moderate rim of deeply basophilic cytoplasm, often containing vacuoles (vacuoles in 9 of 15 cases) Figure 1(1b), $(2 \mathrm{~b})$, and (3b)). The majority of the cases, 13 of 15 $(87 \%)$, showed a predominance (at least $50 \%$ of total red blood cell precursors) of pronormoblasts compared to all erythroid precursors, rendering a potential misinterpretation of an erythroid predominant myelodysplastic syndrome unlikely (Table 2). Ten cases harbored residual erythroid maturation, in which erythroid dysplasia (nuclear budding/blebbing and multinucleation) was clearly present. Morphologic evaluation for granulocytic and megakaryocytic dysplasia was quite limited due to the marked decrease in residual granulopoiesis and megakaryopoiesis. However, megakaryocytes, when present, were often dysplastic (9/15) and characterized by small monolobated forms, micromegakaryocytes (7/15) and/or hyperchromatic forms (4/15). Granulocytic dysplasia could only be reliably assessed in 2 cases.

Bone marrow core biopsy. All cases had adequate bone marrow core biopsies. The bone marrow was uniformly hypercellular for age. Hematoxylin-andeosin-stained sections showed a marked erythroid proliferation with prominent left shift distribution in aggregates and sheets (Figure 1(1c), (2c), and (3c)). The majority of the cases showed a marked predominance of pronormoblasts compared to all erythroid precursors (Table 2). In tissue sections, the pronormoblasts are morphologically remarkable for large round nuclei with stippled chromatin, 1-3 eosinophilic, elongated nucleoli, and a scant to moderate rim of basophilic cytoplasm. On low- and medium-power examination, the pronormoblasts uniformly form large, expansive foci, which on occasion may appear cohesive and/or sinusoidal mimicking a metastatic tumor (not shown). Residual background hematopoiesis is typically markedly decreased due to bone marrow effacement by the erythroid proliferation.

\section{Immunophenotypic Features}

Table 3 summarizes the key immunohistochemical results from the pure erythroid leukemia cases. 


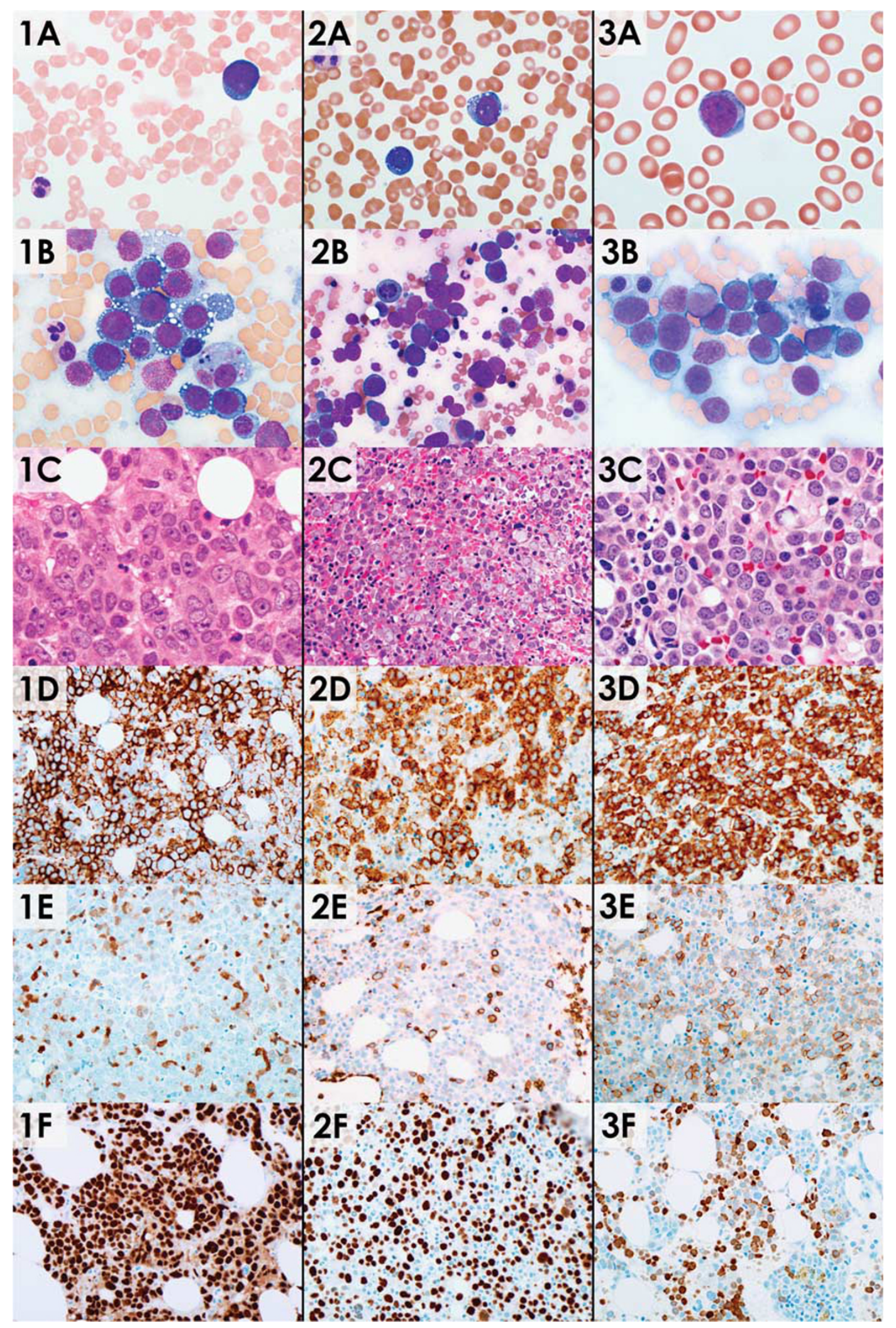

Although not depicted, a variably extensive immunophenotypic assessment was performed not only to definitively demonstrate erythroid lineage but to exclude other mimickers such as carcinoma and lymphoma. This immunophenotypic assessment excludes B-cell lymphoma (CD20, PAX5), T-cell 
Figure 1 This figure is a composite of three separate cases of pure erythroid leukemia and highlight the characteristic cytologic and immunohistochemical features. (1a-f) Case 4; (2a-f) case 6; (3a-f) case 10. (1a) The peripheral blood smear shows significant anemia with an occasional circulating pronormoblast (upper right; $\times 600$ magnification, Wright-Giemsa). (1b) The bone marrow aspirate smear shows a predominant population of highly distinctive abnormal pronormoblasts with discrete, variably sized cytoplasmic vacuoles, round nuclei with multiple nucleoli, and deeply basophilic cytoplasm (×600 magnification, Wright-Giemsa). (1c) High-power examination of the bone marrow core biopsy shows effacement of normal bone marrow by a prominent population of large cells with round-ovoid nuclei, multiple basophilic nucleoli, and moderate eosinophilic cytoplasm (×600 magnification, H\&E). (1d) E-cadherin immunohistochemistry highlights the prominent pronormoblastic proliferation with strong membrane staining ( $\times 400$ magnification, E-cadherin immunohistochemistry). (1e) Hemoglobin is weakly positive in a small subset of the pronormoblasts with more readily identifiable positivity in the more mature erythroid forms (×400 magnification, hemoglobin immunohistochemistry). (1f) TP53 immunohistochemistry shows uniform strong nuclear positivity in the pronormoblastic cells ( $\times 400$ magnification, TP53 immunohistochemistry). (2a) The peripheral blood smear shows moderate anemia with two circulating pronormoblasts with distinctive cytoplasmic vacuoles (middle; $\times 600$ magnification, WrightGiemsa). (2b) The bone marrow aspirate smear shows a prominent erythroid precursor population composed primarily of pronormoblasts with round nuclei and deeply basophilic cytoplasm. This smear shows the occasional phenomenon of numerous broken cells, which may impart difficulty in cellular cytologic assessment and differential counts ( $\times 400$ magnification, Wright-Giemsa). (2c) Medium-power examination of the bone marrow core biopsy shows a prominent interstitial population of large cells with round-ovoid nuclei, multiple basophilic nucleoli, and moderate eosinophilic cytoplasm. Occasional interspersed residual more mature erythroid cells are also noted (middle left; $\times 1000$ magnification, H\&E). (2d) CD71 immunohistochemistry highlights the prominent pronormoblastic proliferation with strong membrane staining with minimal staining of the more mature few residual erythroid precursors $(\times 400$ magnification, CD71 immunohistochemistry). (2e) Glycophorin A stains a rare pronormoblast with more readily identifiable positivity in the more mature erythroid forms ( $\times 400$ magnification, glycophorin A immunohistochemistry). (2f) TP53 immunohistochemistry shows uniform strong nuclear positivity in the pronormoblastic cells ( $\times 400$ magnification, TP53 immunohistochemistry). (3a) The peripheral blood smear shows pancytopenia with a rare circulating pronormoblast with deep blue cytoplasm ( $\times 1000$ magnification, Wright-Giemsa). (3b) The bone marrow aspirate smear shows a prominent erythroid precursor population composed primarily of pronormoblasts with round nuclei, conspicuously stippled chromatin, and deeply basophilic cytoplasm ( $\times 600$ magnification, Wright-Giemsa). (3c) High-power examination of the bone marrow core biopsy shows a prominent sheet-like proliferation of large cells with round-ovoid nuclei, stippled chromatin, occasional small nucleoli, and moderate eosinophilic cytoplasm. Minimal residual normal hematopoiesis is present (middle left; $\times 400$ magnification, H\&E). (3d) CD71 immunohistochemistry shows uniform strong membrane positivity in the pronormoblastic cells $(\times 400$ magnification, CD71 immunohistochemistry). (3e) CD117 immunohistochemistry highlights the pronormoblastic proliferation with typical predominantly weak membrane staining ( $\times 400$ magnification, CD117 immunohistochemistry). (3f) Hemoglobin stains a decent proportion of the pronormoblasts in this case making a determination of erythroid lineage straightforward ( $\times 400$ magnification, hemoglobin immunohistochemistry).

lymphoma (CD3), myeloid/monocytic lineage (myeloperoxidase (MPO) CD13, CD33, and lysozyme), megakaryocytic lineage (CD61), carcinoma (OSCAR and various cytokeratins), and melanoma (S100).

Immunohistochemistry. In general, the demonstration of erythroid lineage in the neoplastic cells resulted from a combination of positive erythroidassociated or erythroid-specific markers, and absence of non-erythroid lineage markers. An immunophenotypic combination of CD71, CD117, E-cadherin, hemoglobin, OSCAR, and myeloperoxidase are, at a minimum, useful in this regard. The pronormoblasts were positive for CD71 (14/14), CD117 (13/14), and E-cadherin (12/13; Table 3) (Figure 1, 1d, 2d, 3d-3e). Of the erythroid-specific markers (glycophorin A and hemoglobin), the pronormoblastic proliferation was in general less positive, demonstrating only weak and subset positivity (Figure 1, 1e, 2e, 3f) (Table 3). CD61 was performed in 12 of the 15 cases and showed markedly decreased megakaryocytes in 10 of the 12 cases with no co-expression on the proerythroblasts. CD34 was negative in 13 of 14 cases with dim partial positivity in 1 case. In all, 7/7 cases were negative for myeloperoxidase. TP53 immunohistochemistry was positive in $9 / 9$ tested cases. This positivity was seen as strong, diffuse nuclear staining in the tumor cells (Figure 1, 1f and 2f).

Flow cytometric immunophenotyping. Flow cytometry was performed in 13 of 15 cases. The findings with regard to the precursor erythroid population were predominantly non-contributory due to hemodilution and/or loss of the precursor erythroid population due to the red blood lysis procedure. However, in 5 cases the presence or absence of CD34 and/or CD117 expression was reliably determined by flow cytometry (Table 3). In addition, bright CD36 positivity, as expected, was demonstrated in the cases where the cells of interest were present.

With regard to a myeloblast population, flow cytometry was able to identify either absence or a small percentage of residual myeloid blasts. The immunophenotypic characteristics of the myeloblasts were quite typical, including expression of CD34, CD117, and the myeloid-associated antigens CD13 and/or CD33 in 7 of the 8 cases with discernible myeloblasts. Table 2 highlights the immunophenotypic features of the myeloblast population.

\section{Cytogenetic and FISH Findings}

All 15 cases demonstrated a complex karyotype of which $12(80 \%)$ were markedly complex (>10 abnormalities). Four cases had hyperdiploid karyotypes, and 9 had hypodiploid karyotypes (Figure 2). Many cases harbored classic myeloid-associated abnormalities, such as del(5q) (9/15, 60\%) and/or $-7 / \operatorname{del}(7 q)(5 / 15,33 \%)$. Abnormalities involving chromosome 17 were also frequent, most commonly monosomy $17(8 / 15,53 \%)$ and uncommonly loss/ abnormalities of $17 p(1 / 15,7 \%)$. Six of our 9 cases 




Figure 2 Abnormal, highly complex, karyotypes typify de novo pure erythroid leukemia. (2a) Representative karyogram from case 4 showing a complex hyperdiploid karyotype harboring del(5q) and a 7 p11.2 rearrangements, which likely represents a homogeneously staining region (hsr) (G-banding). (2b) Representative karyogram from case 5 showing a complex karyotype with a 7 p11.2 rearrangement, which likely represents a homogeneously staining region (hsr) (G-banding). (2c) Representative karyogram from case 6 showing a complex hypodiploid karyotype with whole-arm rearrangement and two homogeneously staining regions (G-banding). (2d) Representative karyogram from case 8 showing a complex hypotetraploid karyotype with whole-arm rearrangement and 7p11.2 homogeneously staining region (G-banding).

positive for p53 by immunohistochemistry showed monosomy 17 or $17 p$ deletion/abnormality by karyotype. TP53 FISH was performed in 5 of 15 cases $(3,7,8,10$, and 15) and 3 showed a TP53 deletion (3, 7, and 8). A few cases showed $7 p$ abnormalities (4/15, 27\%) and/or nonrecurrent homogeneously staining regions (4/15, 27\%; Figure 2). An unbalanced whole-arm rearrangement was observed in 4/15 (27\%) cases (Figure 2).

\section{Discussion}

De novo pure erythroid leukemia is a rare entity, representing $<1 \%$ of all acute myeloid leukemia cases in our experience. This cohort, the largest to date, to our knowledge, provides a comprehensive and detailed assessment of the clinicopathologic and cytogenetic features of 15 de novo pure erythroid leukemia cases. This cohort is distinct from other large, inclusive, published series in that secondary forms of pure erythroid leukemia and cases of erythroleukemia (erythroid/myeloid type) are not included. ${ }^{7,12,15}$ This study confirms that pure erythroid leukemia is indeed an uncommon diagnosis, presents a veritable diagnostic challenge, is associated invariably with a complex (often highly complex) karyotype, and has very aggressive clinical course. Given these features, an extensive workup, including clinical history, laboratory studies, morphology, complete immunophenotyping, and cytogenetics is typically required to establish the diagnosis with confidence. Important clues supporting the diagnosis, after meeting revised fourth edition World Health Organization classification criteria, include circulating pronormoblasts in the absence of other intermediate erythroid forms, predominance of pronormoblasts in the aspirate/ biopsy, clear-cut background dysplasia (which is typically difficult to assess in the granulocytic and megakaryocytic lineages given the erythroid 
predominance), immunophenotypic exclusion of non-hematopoietic entities, p53 immunohistochemical positivity, and a highly complex karyotype, which may or may not contain typical myeloidassociated abnormalities.

Morphologically, the diagnosis of pure erythroid leukemia may also be difficult in the setting of a suboptimal or inadequate bone marrow aspirate specimen. In such circumstances, the bone marrow aspirate differential count may not meet the revised fourth edition World Health Organization classification cutoff for a diagnosis of pure erythroid leukemia, rendering the possibility of misinterpretation as a myelodysplastic syndrome likely. Thus, it is critical to evaluate a touch preparation and/or core biopsy for complete interpretation. Indeed, the extent of leukemic infiltration by pronormoblasts is often more evident on the bone marrow core biopsy, frequently demonstrating diffuse effacement of normal hematopoietic architecture., ${ }^{1,2,7,14}$ Immunohistochemistry, in these situations, may be useful not only in delineating erythroid lineage but also in providing an overall quantification of leukemic involvement.

Immunophenotypic studies are necessary, but can be challenging, in the evaluation of pure erythroid leukemia. First, the lack of specificity of CD117, CD71, and E-cadherin to discriminate neoplastic from non-neoplastic erythroid proliferations often requires utilization of extended immunohistochemical panels to exclude other diagnostic possibilities such as other types of acute myeloid leukemia, lymphoma, and various carcinomas. Markers more useful in this regard include alpha-hemoglobin stabilizing protein, ferritin $\mathrm{H}$, and others. ${ }^{24-29}$ Alpha-hemoglobin stabilizing protein has been proposed as a more specific marker for erythroid precursors in pure erythroid leukemia, as compared to CD71 and glycophorin A. ${ }^{24,26}$ Ferritin $\mathrm{H}$ is a specific marker for early erythroid precursors and macrophages, and is typically negative in more mature erythroid forms. ${ }^{27}$ Second, when a pure erythroid leukemia case shows positivity for either of the erythroid-specific markers, glycophorin A or hemoglobin, the staining is often weak or present only in a small subset of the pronormoblasts. Thus, this requires the interpreter to be confident that it is true staining, not background/artifact, to establish erythroid lineage. This a finding that has been previously observed and reported. ${ }^{14}$ In contrast to glycophorin A and hemoglobin A, which are often absent in early pronormoblasts or show only variable staining, CD71 is typically distinctly positive in early erythroid precursors. ${ }^{25}$ On the basis of institutional resources and availability of immunostains, one may have more or less access to the more specific markers. Third, as erythroid progeny arise from bipotent megakaryocyte-erythrocyte progenitors, occasional cases of pure erythroid leukemia may show partial expression of antigens associated with megakaryocytic differentiation (eg, CD41 and CD61).
In our cohort, we did not detect megakaryocytic antigen expression on the proerythroblasts, although we only performed CD61 immunohistochemistry, which could potentially be limited in sensitivity. Fourth, some cases of pure erythroid leukemia may show expression of myeloid markers, such as CD13 or CD33, or T-cell markers, such as CD2 or CD7, which may cause diagnostic confusion with a myeloid or T-cell process rather than erythroid proliferation. ${ }^{7,29}$ Fifth, we noted a very high rate of immunopositivity for p53 in our series $(100 \%$ in nine tested cases), similar to what has been reported in the literature. ${ }^{12}$ Given that the literature suggests that strong diffuse positivity correlates at a molecular level with a TP53 mutation, ${ }^{30}$ we speculate whether this finding may provide another data point supporting a neoplastic proliferation, as one awaits the cytogenetic results. Of the two cases in our series which had molecular studies and p53 immunohistochemistry, both were positive for both TP53 mutation and protein expression (Table 3 , patients 14 and 15).

Karyotypically, de novo pure erythroid leukemia appears to uniformly demonstrate an abnormal karyotype. The karyotype is generally complex $(\geq 3$ abnormalities), with most cases showing highly complex genetic alterations (>10 abnormalities). From our study and an extensive review of the literature, all de novo pure erythroid leukemia cases exhibit an abnormal karyotype (Table 4). In de novo pure erythroid leukemia, over $95 \%$ of cases show a complex karyotype ( $\geq 3$ abnormalities) with $66 \%$ overall showing a markedly complex karyotype (>10 abnormalities; Table 4). Similar to de novo pure erythroid leukemia, the vast majority of secondary pure erythroid leukemia cases also have an abnormal karyotype (>95\%; Table 4). Wong et al, ${ }^{12}$ Linu et al, ${ }^{13}$ and Bacher et al ${ }^{18}$ in a combined analysis of de novo and secondary pure erythroid leukemia showed $5 / 5,4 / 4$, and $4 / 7$ cases with complex karyotype, respectively. When one compares the cytogenetic alterations between de novo and secondary pure erythroid leukemia, the findings appear similar for the more common abnormalities (Table 4). Thus, in a challenging case when one is considering a diagnosis of pure erythroid leukemia and a normal karyotype is obtained, the diagnosis should be rendered with extreme caution with careful exclusion of other diagnostic entities.

While cases of pure erythroid leukemia, both de novo and secondary, nearly always demonstrate a complex karyotype in the published studies to date, a specific cytogenetic or molecular abnormality has not yet been elucidated. Many, though not all, pure erythroid leukemia cases reported in the literature do demonstrate recurring myeloid-associated cytogenetic abnormalities, such as $\operatorname{del}(5 q),-7 / \operatorname{del}(7 q)$, and $-17 / 17 p$ deletions ${ }^{7,12-17,20}$ (Table 4). Given the highly complex karyotypes, it is often a challenge to do a direct comparison of other, more complicated, genetic rearrangements. 
Table 4 Clinicopathologic findings reported from selected large series of pure erythroid leukemias in the literature since $2000^{\mathrm{a}}$

\begin{tabular}{|c|c|c|c|c|c|c|}
\hline $\begin{array}{l}\text { Author } \\
\text { (reference) }\end{array}$ & $\begin{array}{l}\text { Total number of } \\
\text { reported pure } \\
\text { erythroid leukemia } \\
\text { patients }\end{array}$ & $\begin{array}{l}\text { Total number of de } \\
\text { novo pure erythroid } \\
\text { leukemia patients }\end{array}$ & $\begin{array}{l}\text { Overall median survival of } \\
\text { de novo pure erythroid } \\
\text { leukemia patients (in } \\
\text { months) }\end{array}$ & $\begin{array}{l}\text { Overall median survival of } \\
\text { secondary pure erythroid } \\
\text { leukemia patients (in } \\
\text { months) }\end{array}$ & $\begin{array}{l}\text { De novo pure erythroid } \\
\text { leukemia cytogenetic } \\
\text { karyotypic findings }{ }^{b}\end{array}$ & $\begin{array}{l}\text { Secondary pure } \\
\text { erythroid leukemia } \\
\text { cytogenetic findings }\end{array}$ \\
\hline Park et $a l^{21}$ & 2 & $1^{\mathrm{c}}$ & $3(n=1)$ & $6(n=1)$ & $\begin{array}{l}\text { Abnormal } 100 \% \\
\text { Complex: } 0 / 1 \\
\text { Markedly complex } 0 / 1\end{array}$ & $\begin{array}{l}\text { Abnormal } 100 \% \\
\text { Complex: } 1 / 1 \\
\text { Markedly complex } 0 / 1\end{array}$ \\
\hline Lessard et $a l^{20}$ & 7 & 4 & Not reported & Not reported & $\begin{array}{l}\text { Abnormal } 100 \% \\
\text { Complex: } 4 / 4 \\
\text { Markedly complex: } 1 / 4\end{array}$ & $\begin{array}{l}\text { Abnormal } 100 \% \\
\text { Complex: } 3 / 3 \\
\text { Markedly complex: } 2 / 3\end{array}$ \\
\hline Liu et $a l^{14}$ & 18 & 6 & 3.5 & 3 & $\begin{array}{l}\text { Abnormal } 100 \% \\
\text { Complex: } 5 / 5 \\
\text { Markedly complex: } 3 / 5\end{array}$ & $\begin{array}{l}\text { Abnormal } 100 \% \\
\text { Complex: } 11 / 11 \\
\text { Markedly complex: } 8 / 11\end{array}$ \\
\hline Wang et $a l^{7}$ & 11 & 3 & Not reported & Not reported & $\begin{array}{l}\text { Abnormal } 100 \% \\
\text { Complex: } 3 / 3 \\
\text { Markedly complex: } 2 / 3\end{array}$ & $\begin{array}{l}\text { Abnormal } 100 \% \\
\text { Complex: } 7 / 7 \\
\text { Markedly complex: } 6 / 7\end{array}$ \\
\hline Wong et $a l^{12}$ & 7 & $3^{\mathrm{d}}$ & 6.1 & 1.9 & $\begin{array}{l}\text { Abnormal } 100 \% \text { (two had } \\
\text { no mitoses) } \\
\text { Complex: } 1 / 1 \\
\text { Markedly complex: } 1 / 1\end{array}$ & $\begin{array}{l}\text { Abnormal } 100 \% \\
\text { Complex: } 4 / 4 \\
\text { Markedly complex: } 4 / 4\end{array}$ \\
\hline Li et $a l^{17}$ & 14 & 0 & NA & 2.3 & NA & $\begin{array}{l}\text { Abnormal } 100 \% \\
\text { Complex: } 10 / 10 \\
\text { Markedly complex: } 5 / 10\end{array}$ \\
\hline Park et $a 1^{15}$ & 7 & 3 & 6.6 & 2.1 & $\begin{array}{l}\text { Abnormal } 100 \% \\
\text { Complex: } 3 / 3 \\
\text { Markedly complex: } 1 / 3\end{array}$ & $\begin{array}{l}\text { Abnormal } 100 \% \\
\text { Complex: } 2 / 2 \\
\text { Markedly complex: } 2 / 2\end{array}$ \\
\hline $\begin{array}{l}\text { Montalban- } \\
\text { Bravo et al }{ }^{16}\end{array}$ & 27 & 10 & 3.8 & 2.9 & $\begin{array}{l}\text { Abnormal } 100 \% \\
\text { Complex: } 9 / 10 \\
\text { Markedly complex: } 8 / 10\end{array}$ & $\begin{array}{l}\text { Abnormal } 100 \% \\
\text { Complex: } 16 / 16 \\
\text { Markedly complex: } \\
\text { 14/16 }\end{array}$ \\
\hline Current series & 15 & 15 & 1.4 & NA & $\begin{array}{l}\text { Abnormal } 100 \% \\
\text { Complex: } 15 / 15 \\
\text { Markedly complex: 12/15 }\end{array}$ & NA \\
\hline
\end{tabular}

NA, not applicable.

${ }^{a}$ Certain series were difficult to include because de novo and secondary PEL were not separated for analysis or these cases were grouped with other myeloid malignancies with increased erythroid

precursors. ${ }^{19,13,18}$
${ }^{5}$ Complex karyotype is defined as $\geq 3$ abnormalities) and markedly complex karyotype is defined as $>10$ abnormalities;

${ }^{\mathrm{C}}$ Patient 16 in Park et $\mathrm{al}^{21}$ : no history of chemotherapy/radiotherapy; study does not mention any prior history of chronic myeloid neoplasm.

${ }^{\mathrm{d}}$ One patient has prior therapy. 
Another recurring cytogenetic finding that we and others have observed in pure erythroid leukemia karyotypes is presence of hypodiploidy, hyperdiploidy, or near tetraploidy in a respectable subset of the cases. These findings mirror those that may be seen in non-hematopoietic neoplasms such as carcinoma and sarcoma, and often create diagnostic confusion at a genetic level. The latter is particularly true when a myeloid-associated abnormality such as $-7 / \operatorname{del}(7 q)$ is not observed as part of the complex karyotype. Therefore, it is imperative that the pathologist systematically excludes those diagnoses as part of the workup, particularly as CD71 and E-cadherin lack specificity for erythroid lineage.

Other unique and recurring cytogenetic findings in several of our cases were $7 \mathrm{p}$ abnormalities, wholearm rearrangements, and hsr's (Figure 2), none of which are typically myeloid-associated. Whole-arm rearrangements have been observed in patients with pure erythroid leukemia. ${ }^{31}$ Further molecular assessment of these abnormalities will hopefully provide additional insight into PEL with possible therapeutic implications.

TP53 mutations appear to highly characterize pure erythroid leukemia. Recently, Montalban-Bravo et $a 1^{16}$ demonstrated that pure erythroid leukemia was often characterized by more than one TP53 mutation using targeted sequencing. In their large series, they also noted a high proportion of cases showing co-occurrence of TP53 mutations and a loss of the TP53 genomic region from a deletional event. This suggests that a TP53 abnormality is quite typical of pure erythroid leukemia and certainly has a role in its pathogenesis. In addition, several authors have investigated and reported on the molecular profile of cases of pure erythroid leukemia, along with the previously recognized erythroleukemia (erythroid/ myeloid subtype of acute erythroid leukemia). Similarly to Montalban-Bravo et al, these investigators noted a high incidence of TP53 mutations, but a lower rate of FLT3, NPM1, and NRAS mutations as compared to acute myeloid leukemia, not otherwise specified. . $^{5,16,32}$

The dismal prognosis, median overall survival of 1.4 months in this series, is slightly worse than that reported previously in de novo pure erythroid leukemia: 3.5 months (Liu et $a 1^{14}$ ) and 3.8 months (Montalban-Bravo et $a i^{16}$ Table 4). Park et $a 1^{15}$ showed a slightly longer overall median survival, 6.6 months. When comparing the prognosis of $d e$ novo versus secondary pure erythroid leukemia, the literature suggests that the overall median survival is quite similar with secondary pure erythroid leukemia showing $3,2.9$, and 2.1 months, respectively. ${ }^{14-16} \mathrm{Li}$ et a ${ }^{17}$ examined secondary pure erythroid leukemia and erythroblastic sarcoma, and found a median overall survival of 2.3 months. Other studies have reported a similar poor prognosis in cases of pure erythroid leukemia, although de novo and secondary pure erythroid leukemia cases may not have been separated in the analysis. Bacher et $a 1^{18}$ showed a 2 -year overall survival rate of $30 \%$ for pure erythroid leukemia (three de novo and four secondary cases).

Given the dismal overall median survival for individuals with pure erythroid leukemia, confidence in the final diagnosis and necessary exclusion of other neoplastic myeloid entities is crucial. Important differential diagnostic considerations include myelodysplastic syndrome with an abundance of erythroid elements and acute myeloid leukemia with myelodysplasia-related changes. For myelodysplastic syndrome, several sentinel studies have shown that the determination of the myeloid blast count should be based on total bone marrow nucleated elements rather than as a fraction of the non-erythroid compartment because of the low reproducibility of non-erythroid blast counts and the close biological relationship between erythroleukemia and myelodysplastic syndrome. ${ }^{1-10}$ In the previous 2001 and 2008 World Health Organization classifications, a second subtype of erythroleukemia (erythroid/myeloid type) was recognized in cases with $>50 \%$ erythroid precursors of which $\geq 20 \%$ of the non-erythroid elements consisted of myeloid blasts. As has been shown by Wang and colleagues, it appears that the erythroid/myeloid type of erythroleukemia is biologically and clinically similar to erythroid-rich myelodysplastic syndrome and that small changes in the percent myeloid blasts can result in significant differences in the final diagnostic category. These findings have warranted a change in the revised fourth edition World Health Organization classification. ${ }^{1,2,7-11}$ Specifically, the diagnostic category of erythroleukemia, erythroid/myeloid type has been removed from the revised fourth edition World Health Organization classification and such cases are now categorized as myelodysplastic syndrome. ${ }^{1,2}$ For example, a case with $70 \%$ erythroid precursors, $7 \%$ bone marrow myeloid blasts, and $<1 \%$ circulating blasts would be classified as erythroleukemia (erythroid/myeloid type) in the 2008 World Health Organization classification, and as myelodysplastic syndrome with excess blasts- 1 in whereas in the revised fourth edition World Health Organization classification. For acute myeloid leukemia with myelodysplasia-related changes, while there certainly may be an abundance of erythroid precursors (eg, $>50 \%$ ), $\geq 20 \%$ myeloid blasts (of all nucleated cells) must be present and the other requisite criteria must be met (eg, prior history of myelodysplastic syndrome). ${ }^{1-3}$

Given the focus in the World Health Organization classification on clinicopathologic entities that are clearly defined and clinically distinct, it is not unreasonable to postulate that pure erythroid leukemia be considered a subtype of acute myeloid leukemia with myelodysplasia-related changes rather than acute myeloid leukemia, not otherwise specified. Both of these groups are morphologically and, to some extent, clinically heterogeneous. Currently, pure erythroid leukemia is considered a 
subtype of acute myeloid leukemia, not otherwise specified. While primary pure erythroid leukemia shares many features with acute myeloid leukemia with myelodysplasia-related changes, including variable dysplastic changes, complex karyotype, chromosome 5 and/or 7 abnormalities, and/or TP53 gene alterations, significant differences also exist in that acute myeloid leukemia with myelodysplasia-related changes currently requires $\geq 20 \%$ myeloblasts in the peripheral blood or bone marrow, and includes cases with normal or other nonspecific karyotypes; while pure erythroid leukemia shows a characteristic erythroid proliferation with an abundance of proerythroblasts $(\geq 30 \%)$. As stated in the article by Arber et $a l^{2}$ regarding the revised fourth edition World Health Organization classification, 'the category of acute myeloid leukemia with myelodysplasia-related changes has been refined to better incorporate cases with features suggesting a poor prognosis,' so pure erythroid leukemia would fit if the diagnostic criteria were modified. However, we think the terminology of pure erythroid leukemia would need to be retained, at least at this point, regardless of the overarching category in order to effectively communicate the sufficiently unique morphologic, genetic, and dismal prognosis features of this disorder. Another possibility is to consider pure erythroid leukemia as an entirely separate and distinct clinicopathologic entity. In many studies, it appears that an independent prognostic driver for poor outcome in acute myeloid leukemia is the complex and/or monosomal karyotype, so perhaps in the future the driver of classification and prognostication could be based on genetics.

In conclusion, this study provides detailed clinicopathologic and cytogenetic features of de novo pure erythroid leukemia and a review of the recent published literature. In addition to confirming that pure erythroid leukemia is a rare entity $(<1 \%$ of all acute myeloid leukemias), we emphasize that pure erythroid leukemia can be a challenging diagnosis to make. The diagnostic workup requires an integrated approach and comprehensive assessment, including the demonstration of an abnormal karyotype. Indeed, in the absence of an abnormal, typically complex karyotype, the diagnosis should be reconsidered. On the basis of review of the primary literature, given that the overall median survival and karyotypic findings of de novo pure erythroid leukemia are similar to those reported for secondary pure erythroid leukemia, such a distinction may not be clinically necessary. Additional studies such as next-generation sequencing may facilitate identification of actionable mutations of this highly unusual entity.

\section{Disclosure/conflict of interest}

The authors declare no conflict of interest.

\section{References}

1 Arber DA, Brunning RD, Orazi A et al. Pure erythroid leukemia. In: Swerdlow SH, Campe E, Harris NL, et al (eds). WHO Classification of Tumours of Haematopoietic and Lymphoid Tissues (Revised 4th Edition). IARC: Lyon, France, pp 161-162, 2017.

2 Arber DA, Orazi A, Hasserjian R, et al. The 2016 revision to the World Health Organization classification of myeloid neoplasms and acute leukemia. Blood 2016;127:2391-2405.

3 Arber DA. Revisiting erythroleukemia. Curr Opin Hematol 2017;24:146-151.

4 Liu CJ, Hong YC, Yang CF, et al. Clinicopathologic features and outcome of acute erythroid leukemia based on 2008 revised World Health Organization classification. Leuk Lymphoma 2012;53:289-294.

5 Zuo Z, Medeiros LJ, Chen Z, et al. Acute myeloid leukemia (AML) with erythroid predominance exhibits clinical and molecular characteristics that differ from other types of AML. PLoS ONE 2012;7:e41485.

6 Grossmann V, Bacher U, Haferlach C, et al. Acute erythroid leukemia (AEL) can be separated into distinct prognostic subsets based on cytogenetic and molecular genetic characteristics. Leukemia 2013;27:1940-1943.

7 Wang SA, Hasserjian RP. Acute erythroleukemias, acute megakaryoblastic leukemias, and reactive mimics: a guide to a number of perplexing entities. Am J Clin Pathol 2015;144:44-60.

8 Hasserjian RP, Zuo Z, Garcia C, et al. Acute erythroid leukemia: a reassessment using criteria refined in the 2008 WHO classification. Blood 2010;115:1985-1992.

9 Wang SA, Patel KP, Pozdnyakova O, et al. Acute erythroid leukemia with $<20 \%$ bone marrow blasts is clinically and biologically similar to myelodysplastic syndrome with excess blasts. Mod Pathol 2016;29:1221-1231.

10 Wang W, Wang SA, Medeiros LJ, et al. Pure erythroid leukemia. Am J Hematol 2017;92:292-296.

11 Wang SA, Hasserjian RP. Erythroid proliferations in myeloid neoplasms. Hum Pathol 2012;43:153-164.

12 Wong E, Ling V, Westerman D, et al. How unique is pure erythroid leukaemia? A retrospective analysis of seven cases and review of the literature. J Clin Pathol 2015;68:301-305.

13 Linu JA, Udupa MN, Madhumathi DS, et al. Study of clinical, haematological and cytogenetic profile of patients with acute erythroid leukaemia. Ecancermedicalscience 2017;11:712.

$14 \mathrm{Liu} \mathrm{W}$, Hasserjian RP, Hu Y, et al. Pure erythroid leukemia: a reassessment of the entity using the 2008 World Health Organization classification. Mod Pathol 2011;24:375-383.

15 Park DC, Ozkaya N, Lovitch SB. Acute leukaemia with a pure erythroid phenotype: under-recognized morphological and cytogenetic signatures associated universally with primary refractory disease and a dismal clinical outcome. Histopathology 2017;71:316-321.

16 Montalban-Bravo G, Benton CB, Wang SA, et al. More than 1 TP53 abnormality is a dominant characteristic of pure erythroid leukemia. Blood 2017;129:2584-2587.

17 Li H, Hasserjian RP, Kroft SH, et al. Pure erythroid leukemia and erythroblastic sarcoma evolving from chronic myeloid neoplasms. Am J Clin Pathol 2016;145:538-551.

18 Bacher U, Haferlach C, Alpermann T, et al. Comparison of genetic and clinical aspects in patients with acute myeloid leukemia and myelodysplastic syndromes all 
with more than $50 \%$ of bone marrow erythropoietic cells. Haematologica 2011;96:1284-1292.

19 Domingo-Claros A, Larriba I, Rozman M, et al. Acute erythroid neoplastic proliferations. A biological study based on 62 patients. Haematologica 2002;87:148-153.

20 Lessard M, Struski S, Leymarie V, et al. Cytogenetic study of 75 erythroleukemias. Cancer Genet Cytogenet 2005;163:113-122.

21 Park S, Picard F, Azgui Z, et al. Erythroleukemia: a comparison between the previous FAB approach and the WHO classification. Leuk Res 2002;26:423-429.

22 Morice WG, Kurtin PJ, Hodnefield JM, et al. Predictive value of blood and bone marrow flow cytometry in B-cell lymphoma classification: comparative analysis of flow cytometry and tissue biopsy in 252 patients. Mayo Clin Proc 2008;83:776-785.

23 McGowan-Jordan J, Simons A, Schmid M. (eds). ISCN 2016: An International System for Human Cytogenomic Nomenclature (2016). Karger: Basel, Freiburg, 2016.

24 Raess PW, Paessler ME, Bagg A, et al. alphaHemoglobin-stabilizing protein is a sensitive and specific marker of erythroid precursors. Am J Surg Pathol 2012;36:1538-1547.

25 Dong HY, Wilkes S, Yang H. CD71 is selectively and ubiquitously expressed at high levels in erythroid precursors of all maturation stages: a comparative immunochemical study with glycophorin A and hemoglobin A. Am J Surg Pathol 2011;35:723-732.
$26 \mathrm{Yu} \mathrm{H}$, Pinkus JL, Pinkus GS. alpha-Hemoglobinstabilizing protein: an effective marker for erythroid precursors in bone marrow biopsy specimens. Appl Immunohistochem Mol Morphol 2016;24:51-56.

27 Wang W, Grier DD, Woo J, et al. Ferritin H is a novel marker of early erythroid precursors and macrophages. Histopathology 2013;62:931-940.

28 Rollins-Raval MA, Fuhrer K, Marafioti T, et al. ALDH, CA I, and CD2AP: novel, diagnostically useful immunohistochemical markers to identify erythroid precursors in bone marrow biopsy specimens. Am J Clin Pathol 2012;137:30-38.

29 Hasserjian RP. Erythroleukemia and its differential diagnosis. Surg Pathol Clin 2013;6:641-659.

30 Fernandez-Pol S, Ma L, Ohgami RS, et al. Immunohistochemistry for p53 is a useful tool to identify cases of acute myeloid leukemia with myelodysplasia-related changes that are TP53 mutated, have complex karyotype, and have poor prognosis. Mod Pathol 2017;30: 382-392.

31 Mackinnon RN, Wall M, Zordan A, et al. Genome organization and the role of centromeres in evolution of the erythroleukaemia cell line HEL. Evol Med Public Health 2013;2013:225-240.

32 Cervera N, Carbuccia N, Garnier S, et al. Molecular characterization of acute erythroid leukemia (M6-AML) using targeted next-generation sequencing. Leukemia 2016;30:966-970. 